\section{Fatores associados ao sedentarismo no lazer em idosos, Campinas, São Paulo, Brasil}

\author{
Variables associated with sedentary leisure time \\ in the elderly in Campinas, São Paulo State, Brazil
}

\author{
${ }^{1}$ Faculdade de Ciências \\ Médicas, Universidade \\ Estadual de Campinas, \\ Campinas, Brasil. \\ 2 Faculdade de Saúde \\ Pública, Universidade de \\ São Paulo, São Paulo, Brasil. \\ 3 Faculdade de Medicina \\ de Botucatu, Universidade \\ Estadual Paulista, Botucatu, \\ Brasil. \\ 4 Faculdade de Medicina, \\ Universidade de São Paulo, \\ São Paulo, Brasil. \\ Correspondência \\ M. P. A. Zaitune \\ Departamento de Medicina \\ Preventiva e Social, \\ Faculdade de Ciências \\ Médicas, Universidade \\ Estadual de Campinas. \\ C. P. 6111, Campinas, SP \\ 13083-970, Brasil. \\ mpaula@fcm.unicamp.br
}

\begin{abstract}
The objective of this study was to identify the prevalence of sedentary leisure time (no type of leisure-time exercise once a week or more) among the elderly in the city of Campinas, São Paulo State, Brazil, according to demographic and socioeconomic factors, other health-related behaviors, and the presence of morbidity. This was a population-based cross-sectional study with multiple-stage sampling. Data analysis considered the sample design. Prevalence of sedentary leisure time was $70.9 \%$, and the prevalence ratio was significantly higher than 1.0 for the elderly with lower socioeconomic status (1.31: 1.11-1.55), smokers (1.39: 1.23-1.57), those with common mental disorders (1.20: 1.041.39), and females (1.16: 1.00-1.35). Prevalence of walking was $23.5 \%$, followed by fitness or bodybuilding programs (3.8\%), and swimming or water aerobics (3.6\%). The results show the need to develop global action for health-related behaviors in order for this approach to succeed. Attention should focus on the elderly with common mental disorders and those with lower socioeconomic status to ensure equity in health promotion practices.
\end{abstract}

Leisure Activities; Exercise; Aged; Health Behavior; Health Promotion
Maria Paula do Amaral Zaitune 1 Marilisa Berti de Azevedo Barros 1 Chester Luiz Galvão César ${ }^{2}$

Luana Carandina ${ }^{3}$

Moisés Goldbaum 4

\section{Introdução}

Diversas pesquisas apontam que o sedentarismo, combinado a outros fatores de risco, contribui para a ocorrência de um conjunto de doenças crônicas, como: diabetes, osteoporose, câncer de cólon, de pulmão e de próstata e, sobretudo, doenças cardiovasculares 1,2,3.

Inúmeros benefícios físicos e psicossociais que resultam da prática regular de atividade física são relatados na literatura 2,4,5. Entre eles, estão: o aumento da força muscular, a melhora do condicionamento cardiorrespiratório, a redução de gordura, o aumento da densidade óssea, a melhora do humor e da auto-estima e a redução da ansiedade e da depressão 6 .

Os benefícios da prática de atividade física não se restringem ao campo fisico-funcional e mental dos indivíduos, mas repercutem também na dimensão social, melhorando o desempenho funcional, mantendo e promovendo a independência e a autonomia daqueles que envelhecem. Especialmente entre os idosos, é constatado que a prática de atividade física diminui o risco de institucionalização e o uso de serviços de saúde e de medicamentos 7 .

Pelas evidências acumuladas quanto aos efeitos benéficos que produz, a atividade física vem sendo crescentemente inserida em programas de promoção de hábitos saudáveis de vida, de prevenção e mesmo de controle de doenças 8 . 
Tem sido verificado que vários fatores estão associados ao sedentarismo: nível sócio-econômico, gênero e contextos ambientais e sociais como o acesso a espaços físicos atrativos, menor tráfego de carros etc 9 .

Alguns autores elegem o exercício praticado no lazer para estimar a prevalência de ativos ou de fisicamente inativos por considerarem estas informações mais diretas e fáceis de obter que as relativas às atividades realizadas no exercício ocupacional ou no trabalho doméstico 10. Visto que a população idosa, em sua maioria, não se encontra mais engajada em atividades econômicas, avalia-se que o sedentarismo no lazer seja um indicador adequado de inatividade neste segmento da população.

Sob esta ótica, estabeleceu-se como objetivo deste estudo conhecer a prevalência de sedentarismo durante o lazer em idosos residentes do Município de Campinas, São Paulo, Brasil, analisando-a segundo fatores demográficos e sócioeconômicos, presença de morbidade e outros comportamentos relacionados à saúde.

\section{Metodologia}

\section{População de estudo}

Trata-se de pesquisa de corte transversal, de base populacional, que utilizou dados do estudo multicêntrico Inquérito de Saúde de Base Populacional no Estado de São Paulo (ISA-SP), realizado em quatro áreas do Estado, sendo dois municípios do interior (Botucatu e Campinas), a subprefeitura do Butantã, pertencente ao Município de São Paulo e uma área formada por três municípios da região sudoeste da Grande São Paulo (Taboão da Serra, Embu e Itapecerica da Serra). A pesquisa foi realizada por três universidades públicas do Estado (Universidade de São Paulo - USP, Universidade Estadual Paulista - UNESP e Universidade Estadual de Campinas - UNICAMP) e contou com a parceria da Secretaria Estadual da Saúde de São Paulo 11.

A amostragem foi feita por conglomerados, estratificada e em múltiplos estágios. Com base nos dados do Instituto Brasileiro de Geografia e Estatística (IBGE), os setores censitários foram classificados e agrupados em três estratos segundo o percentual de chefes de família com nível universitário: menos de $5 \%$, de 5 a $25 \%$ e mais de $25 \%$. Em cada área, de cada estrato, foram sorteados dez setores censitários, dos quais foram sorteados domicílios e em cada domicílio, foram entrevistados indivíduos, segundo domínios definidos de idade e sexo. Os domínios amostrais foram: menor de um ano, dois a 11 anos, 12 a 19 anos masculino, 12 a 19 anos feminino, 20 a 59 anos masculino, 20 a 59 anos feminino, 60 anos e mais masculino e 60 anos e mais feminino. Foi estimado um tamanho mínimo de amostra de 196 pessoas para cada domínio de idade e sexo, tendo por base a estimativa de uma prevalência de $50 \%$, efeito de delineamento de 2, erro máximo de 0,07 e nível de $95 \%$ de confiança. Considerando uma possível perda de $20 \%$ foram selecionados 250 indivíduos em cada domínio.

As informações foram obtidas por meio de questionário aplicado por entrevistadores treinados diretamente à pessoa selecionada, nos anos de 2001 e 2002. O questionário foi composto principalmente por questões fechadas, organizadas em blocos temáticos: condições de vida, estilo de vida, percepção e qualidade de saúde, morbidade crônica referida, uso de serviços de saúde e consumo de medicamentos, entre outros.

Para o presente estudo, foram analisados os dados apenas do Município de Campinas, relativos aos dois domínios de 60 anos e mais, masculino e feminino.

As variáveis incluídas no presente estudo foram:

- Sedentarismo no lazer: foi considerado sedentário o indivíduo que referiu não praticar qualquer esporte ou exercício físico pelo menos uma vez na semana. A pergunta feita ao entrevistado foi "Pratica regularmente, pelo menos uma vez por semana, algum esporte ou exercício físico? Se sim, qual?". Esta questão era semi-aberta e permitia mais de uma resposta quanto ao tipo de esporte ou exercício físico praticado;

- Demográficas: sexo, idade, cor, estado conjugal, condição de chefia na família, número de moradores no domicílio, naturalidade e religião; - Sócio-econômicas: escolaridade, renda familiar mensal per capita (em salários mínimos) e atividade ocupacional;

- Comportamentos relacionados à saúde: freqüência semanal de ingestão de bebida alcoólica, dependência alcoólica avaliada por meio do teste CAGE 12 e hábito de fumar;

- Morbidades: transtorno mental comum avaliado com base no Self Reporting Questionnaire (SRQ-20), com ponto de corte 7/8 13, número de morbidades crônicas referidas, auto-avaliação da saúde e índice de massa corporal $\left(\mathrm{IMC}=\mathrm{kg} / \mathrm{m}^{2}\right.$ ) calculado com dados de peso e altura referidos, o que é amplamente utilizado e que tem sido considerado válido para estudos populacionais 14 . O IMC dos indivíduos foi classificado como: normal (IMC até $24,9 \mathrm{~kg} / \mathrm{m}^{2}$ ), sobrepeso (IMC $\geq 25$ a $29,9 \mathrm{~kg} / \mathrm{m}^{2}$ ) e obeso (IMC $\geq 30,0 \mathrm{~kg} / \mathrm{m}^{2}$ ). 


\section{Análise dos dados}

As informações obtidas nas entrevistas foram digitadas em banco de dados desenvolvido com o uso do programa Epi Info 6, versão 6.04b (Centers for Disease Control and Prevention, Atlanta, Estados Unidos). A associação entre as diversas variáveis e o sedentarismo no lazer foi verificada pelo teste 2 com nível de significância de 5\%. Foram calculadas razões de prevalência e intervalos de confiança de 95\% por meio de regressão de Poisson. Para o modelo múltiplo, também realizado pela regressão de Poisson, foram consideradas as variáveis que tiveram valor de $\mathrm{p}<0,2015$ na análise bivariada (idade, sexo, situação conjugal, cor, religião, número de moradores no domicílio, naturalidade, renda, escolaridade, freqüência de ingestão de bebida alcoólica, hábito de fumar, índice de massa corporal, número de doenças crônicas, transtorno mental comum, auto-avaliação da saúde), e permaneceram no modelo as variáveis com $p<0,05$. Dado que o tamanho da amostra não é muito grande, para entrada no modelo de regressão foram dicotomizadas as variáveis: escolaridade ( 0 a 4 anos e 5 e mais) e hábito de fumar (fumante e não fumante). Todas as análises, feitas com o programa Stata 7.0 (Stata Corporation, College Station, Estados Unidos), incorporaram as ponderações necessárias ao desenho amostral.

O projeto deste estudo foi aprovado pelo Comitê de Ética em Pesquisa da Faculdade de Ciências Médicas da UNICAMP sob o parecer no ${ }^{\circ}$ 369/2000.

\section{Resultados}

Nos domicílios sorteados do Município de Campinas de forma a encontrar um número satisfatório de indivíduos de cada domínio de idade e sexo, foram encontrados 469 idosos que deveriam participar da pesquisa, porém, registraram-se 9,2\% de recusas $(\mathrm{n}=43)$.

Portanto, para este estudo foram analisadas as informações de 426 idosos (idade igual ou superior a 60 anos), não institucionalizados, residentes na área urbana do Município de Campinas, dos quais 208 eram do sexo feminino e 186 tinham idade superior a 70 anos. A média de idade foi de 69,8 anos (desvio padrão $=7,4$ ).

A prevalência de sedentarismo no lazer em idosos no Município de Campinas foi 70,9\% (IC95\%: $65,0 \%-76,2 \%$ ), sendo $65,8 \%$ nos homens e $74,7 \%$ nas mulheres (Tabela 1 ).

Observou-se que idosos sem cônjuge, evangélicos, que moram com cinco ou mais pessoas no domicílio e de cor não-branca apresentaram prevalência de sedentarismo significativamente maior que as respectivas categorias de referência (Tabela 1).

Em relação aos fatores sócio-econômicos, o sedentarismo mostrou-se mais freqüente, de forma estatisticamente significante, nos idosos com menor renda familiar per capita e menor escolaridade (Tabela 2).

Ao analisar a prevalência de sedentarismo segundo estilo vida, morbidades e auto-avaliação da saúde (Tabela 3) verificou-se ser o sedentarismo mais prevalente, de forma estatisticamente significante, entre os idosos fumantes, obesos, com transtorno mental comum e que percebem sua saúde como ruim.

Na análise múltipla, usando-se a regressão de Poisson (Tabela 4), observou-se que menor renda, ser fumante, ter transtorno mental e ser do sexo feminino revelam-se associadas positivamente ao sedentarismo no lazer.

A Tabela 5 apresenta a prevalência das modalidades de exercício realizadas pelos idosos do município. Dentre elas, a caminhada foi a atividade mais freqüente, sendo praticada por $23,5 \%$ dos idosos, seguida por ginástica ou musculação $(3,8 \%)$ e por natação ou hidroginástica (3,6\%). Pode-se observar também que a prática de natação ou hidroginástica foi mais prevalente nas mulheres. Verificou-se que os idosos de maior escolaridade apresentam maior prevalência da prática de todas as modalidades de exercício comparativamente aos demais. De igual modo, idosos com renda igual ou superior a 2,5 salários mínimos também apresentam maior prevalência da prática de todos os tipos de exercício, embora a diferença seja estatisticamente significante apenas para a caminhada. Em relação à faixa etária, não houve diferença entre as diversas modalidades para as faixas de 60 a 69 anos e 70 anos e mais, ficando o grupo "outras modalidades", que inclui: vôlei, futebol, bicicleta e outras, no limiar de significância estatística.

\section{Discussão}

Este estudo refere-se aos dados da população de 60 anos e mais obtidos por meio de inquérito de base populacional realizado em Campinas, fornecendo informações do sedentarismo no lazer e dos fatores associados nesse subgrupo etário da população. Os principais resultados obtidos foram: prevalência de sedentarismo no lazer elevada, porém dentro do padrão encontrado na literatura e razões de prevalências significativamente superiores entre os idosos de menor renda, tabagistas, do sexo feminino e 
Prevalência de sedentarismo no lazer segundo variáveis sócio-demográficas em pessoas com 60 anos ou mais. Campinas, São Paulo, Brasil, 2001-2002.

\begin{tabular}{|c|c|c|c|c|}
\hline Variáveis e categorias & $\mathbf{N}$ * & Sedentarismo no lazer (\%) & IC95\% & RP (IC95\%) \\
\hline Sexo & & 0,112 ** & & \\
\hline Masculino & 218 & 65,8 & $59,6-71,5$ & 1,00 \\
\hline Feminino & 208 & 74,7 & $65,3-82,2$ & $1,14(0,98-1,32)$ \\
\hline Total & 426 & 70,9 & $65,0-76,2$ & \\
\hline Faixa etária (em anos) & & $0,106 * \star$ & & \\
\hline $60-69$ & 240 & 71,0 & $63,8-77,2$ & 1,00 \\
\hline $70-79$ & 139 & 66,3 & $58,2-73,6$ & $0,93(0,82-1,06)$ \\
\hline 80 e + & 47 & 83,8 & $64,9-93,6$ & $1,18(1,00-1,39)$ \\
\hline Cor & & 0,058 ** & & \\
\hline Branca & 360 & 68,8 & $62,6-74,5$ & 1,00 \\
\hline Não-branca & 65 & 82,7 & $69,1-91,2$ & $1,20(1,03-1,41)$ \\
\hline Situação conjugal & & 0,013 ** & & \\
\hline Com cônjuge & 266 & 67,2 & $61,2-72,8$ & 1,00 \\
\hline Sem cônjuge & 160 & 76,2 & $68,8-82,3$ & $1,13(1,03-1,24)$ \\
\hline Naturalidade & & 0,086 ** & & \\
\hline Estado de São Paulo & 283 & 67,8 & $61,5-73,5$ & 1,00 \\
\hline Outros Estados & 142 & 76,8 & $66,4-84,7$ & $1,13(0,99-1,29)$ \\
\hline Religião & & 0,052 ** & & \\
\hline Católica & 331 & 69,4 & $64,2-74,2$ & 1,00 \\
\hline Evangélica & 54 & 81,8 & $69,1-90,1$ & $1,18(1,05-1,32)$ \\
\hline Outras/Sem religião & 41 & 67,4 & $52,4-79,4$ & $0,97(0,81-1,16)$ \\
\hline Número de moradores no domicílio & & $0,011 * \star$ & & \\
\hline $1-4$ & 344 & 68,1 & $61,6-73,9$ & 1,00 \\
\hline 5 ou + & 82 & 82,5 & $73,1-89,1$ & $1,21(1,06-1,38)$ \\
\hline Condição de chefia & & 0,286 ** & & \\
\hline Não-chefe & 141 & 74,3 & $63,9-82,5$ & 1,00 \\
\hline Chefe & 285 & 68,9 & $62,8-74,5$ & $0,93(0,81-1,06)$ \\
\hline
\end{tabular}

* Número de indivíduos na amostra não ponderada;

** Valor $p$ do teste $\chi^{2}$

com transtorno mental comum, quando comparados às respectivas categorias de referência.

O exercício praticado no lazer é um dos componentes da atividade física global, não avaliando a atividade desenvolvida em outros contextos, como no trabalho, nos deslocamentos e nas atividades domésticas. Entretanto, o estudo do sedentarismo no lazer pode ser um indicador adequado da inatividade física em idosos que, em sua maioria, não estão mais inseridos em atividades ocupacionais. Além disso, a prática de exercício no período de recreação ou lazer constitui um indicador de incorporação de estilos de vida mais saudáveis e compõe-se de práticas que podem ser promovidas pelos programas de setores sociais e dos serviços de saúde. Pela definição de sedentarismo no lazer adotada neste estudo, é preciso lembrar que aqueles indivíduos que pra- ticam algum exercício físico ao menos uma vez na semana não foram considerados sedentários, embora possam ser insuficientemente ativos.

Ainda que não contemplado neste estudo, outras pesquisas apontam para a importância da atividade física realizada no contexto doméstico na melhora e manutenção da capacidade funcional dos idosos 16 .

Uma das limitações do presente estudo é a obtenção da informação referida da prática de exercício por meio de questionário e não pelo uso de equipamentos de mensuração. Há pesquisas apontando que a informação referida pode subestimar 3 ou superestimar 17 a prevalência de ativos. A atividade física, sendo um comportamento socialmente desejável, tende a ser superestimada nas pesquisas, assim como as condutas indesejáveis tendem a ser subesti- 
Prevalência de sedentarismo no lazer segundo variáveis sócio-econômicas em pessoas com 60 anos ou mais. Campinas, São Paulo, Brasil, 2001-2002.

\begin{tabular}{|c|c|c|c|c|}
\hline Variáveis e categorias & $N$ * & Sedentarismo no lazer (\%) & IC95\% & RP (IC95\%) \\
\hline Escolaridade (em anos) & & $0,003 * *$ & & \\
\hline $9 e+$ & 89 & 55,9 & $42,6-68,3$ & 1,00 \\
\hline $5-8$ & 32 & 67,9 & $52,3-80,3$ & $1,22(0,88-1,67)$ \\
\hline $0-4$ & 302 & 75,5 & $69,2-80,9$ & $1,35(1,07-1,70)$ \\
\hline Renda per capita (em salários mínimos) & & $0,001 * \star$ & & \\
\hline$\geq 2,5$ & 202 & 59,8 & $51,6-67,5$ & 1,00 \\
\hline$<2,5$ & 224 & 80,5 & $73,5-86,0$ & $1,35(1,15-1,58)$ \\
\hline Atividade econômica & & $0,515 * \star$ & & \\
\hline Não & 320 & 70,1 & $62,2-76,9$ & 1,00 \\
\hline Sim & 106 & 73,8 & $65,6-80,6$ & $1,05(0,90-1,23)$ \\
\hline
\end{tabular}

* Número de indivíduos na amostra não ponderada;

** Valor $p$ do teste $\chi^{2}$.

Tabela 3

Prevalência de sedentarismo no lazer segundo estilo de vida, morbidade e auto-avaliação em saúde em pessoas com 60 anos ou mais. Campinas, São Paulo, Brasil, 2001-2002.

\begin{tabular}{|c|c|c|c|c|}
\hline Variáveis e categorias & $N$ * & Sedentarismo no lazer (\%) & IC95\% & RP (IC95\%) \\
\hline Hábito de fumar & & $0,005 * *$ & & \\
\hline Não-fumante & 238 & 68,6 & $59,9-76,2$ & 1,00 \\
\hline Fumante & 47 & 93,8 & $81,7-98,1$ & $1,36(1,19-1,57)$ \\
\hline Ex-fumante & 140 & 68,0 & $59,9-75,1$ & $0,99(0,85-1,16)$ \\
\hline Dependência de bebida alcoólica & & $0,386 \star \star$ & & \\
\hline Não dependente & 402 & 70,3 & $64,2-75,9$ & 1,00 \\
\hline Dependente & 18 & 79,8 & $55,2-92,7$ & $1,14(0,88-1,46)$ \\
\hline $\begin{array}{l}\text { Freqüência de ingestão de bebida } \\
\text { alcoólica (por semana) }\end{array}$ & & $0,115 * \star$ & & \\
\hline$\geq 2$ vezes & 80 & 63,2 & $53,5-71,9$ & 1,00 \\
\hline$<2$ vezes/Não bebe álcool & 346 & 72,4 & $65,5-78,4$ & $1,15(0,96-1,37)$ \\
\hline Índice de massa corporal & & 0,008 ** & & \\
\hline Normal & 190 & 74,0 & $65,6-81,0$ & 1,00 \\
\hline Sobrepeso & 141 & 60,5 & $50,5-69,8$ & $0,82(0,68-0,98)$ \\
\hline Obeso & 95 & 79,1 & $70,0-86,0$ & $1,07(0,94-1,22)$ \\
\hline Número de doenças crônicas & & $0,156 * \star$ & & \\
\hline $0-4$ & 346 & 68,8 & $62,2-74,8$ & 1,00 \\
\hline 5 ou + & 68 & 78,4 & $65,3-87,5$ & $1,14(0,97-1,34)$ \\
\hline Transtorno mental comum & & $0,001 * \star$ & & \\
\hline Ausente & 324 & 66,2 & $59,2-72,7$ & 1,00 \\
\hline Presente & 79 & 84,9 & $75,8-91,0$ & $1,28(1,13-1,45)$ \\
\hline Auto-avaliação da saúde & & 0,015 ** & & \\
\hline Excelente/Muito boa & 124 & 59,0 & $48,4-68,8$ & 1,00 \\
\hline Boa/Ruim/Muito ruim & 277 & 74,8 & $67,0-81,3$ & $1,27(1,04-1,55)$ \\
\hline
\end{tabular}

* Número de indivíduos na amostra não ponderada;

** Valor $p$ do teste $\chi^{2}$. 
Tabela 4.

Modelo de regressão múltipla de Poisson: variáveis associadas ao sedentarismo no lazer em idosos.

Campinas, São Paulo, Brasil, 2001-2002.

\begin{tabular}{|c|c|c|}
\hline Variáveis e categorias & RP bruta (IC95\%) & RP ajustada * (IC95\%) \\
\hline \multicolumn{3}{|c|}{ Renda per capita (em salários mínimos) } \\
\hline$\geq 2,5$ & 1,00 & 1,00 \\
\hline$<2,5$ & $1,35(1,15-1,58)$ & $1,31(1,11-1,55)$ \\
\hline \multicolumn{3}{|l|}{ Sexo } \\
\hline Masculino & 1,00 & 1,00 \\
\hline Feminino & $1,14(0,98-1,32)$ & $1,16(1,00-1,35)$ \\
\hline \multicolumn{3}{|l|}{ Hábito de fumar } \\
\hline Não-fumante & 1,00 & 1,00 \\
\hline Fumante & $1,37(1,21-1,55)$ & $1,39(1,23-1,57)$ \\
\hline \multicolumn{3}{|l|}{ Transtorno mental comum } \\
\hline Ausente & 1,00 & 1,00 \\
\hline Presente & $1,28(1,13-1,45)$ & $1,20(1,04-1,39)$ \\
\hline
\end{tabular}

* Ajustada pelo método de regressão de Poisson para as demais variáveis da tabela.

Prevalência da prática de exercício por idosos, segundo sexo, escolaridade, renda e faixa etária.

Campinas, São Paulo, Brasil, 2001-2002.

\begin{tabular}{|c|c|c|c|c|c|c|c|c|}
\hline \multirow[t]{4}{*}{ Variáveis } & \multicolumn{8}{|c|}{ Modalidades de exercício } \\
\hline & \multirow{2}{*}{\multicolumn{2}{|c|}{ Caminhada }} & \multirow{2}{*}{\multicolumn{2}{|c|}{$\begin{array}{c}\text { Natação/ } \\
\text { Hidroginástica }\end{array}$}} & \multirow{2}{*}{\multicolumn{2}{|c|}{$\begin{array}{l}\text { Ginástica/ } \\
\text { Musculação }\end{array}$}} & \multirow{2}{*}{\multicolumn{2}{|c|}{ Outras * }} \\
\hline & & & & & & & & \\
\hline & $\mathbf{n}$ & $\%$ & $\mathrm{n}$ & $\%$ & $\mathrm{n}$ & $\%$ & $\mathbf{n}$ & $\%$ \\
\hline \multicolumn{9}{|l|}{ Sexo } \\
\hline Masculino & 62 & 28,5 & 2 & 0,9 & 6 & 2,8 & 9 & 4,0 \\
\hline Feminino & 43 & 19,9 & 10 & 4,5 & 10 & 4,6 & 5 & 2,3 \\
\hline Total & 105 & 23,5 & 12 & 3,0 & 16 & 3,8 & 14 & 3,1 \\
\hline Valor $\mathrm{p}$ & & 0,104 & & 0,013 & & 0,255 & & 0,363 \\
\hline \multicolumn{9}{|c|}{ Escolaridade (em anos) } \\
\hline $0-4$ & 66 & 20,5 & 4 & 1,5 & 7 & 2,5 & 9 & 2,7 \\
\hline 5 e + & 39 & 32,1 & 8 & 7,0 & 8 & 6,6 & 5 & 4,0 \\
\hline Valor $p$ & & 0,021 & & 0,021 & & 0,033 & & 0,421 \\
\hline \multicolumn{9}{|c|}{ Renda per capita (em salários mínimos) } \\
\hline$<2,5$ & 37 & 15,3 & 4 & 1,9 & 6 & 2,8 & 7 & 2,9 \\
\hline$\geq 2,5$ & 68 & 33,0 & 8 & 4,2 & 10 & 5,0 & 7 & 3,2 \\
\hline Valor $\mathrm{p}$ & & 0,001 & & 0,202 & & 0,2973 & & 0,851 \\
\hline \multicolumn{9}{|c|}{ Faixa etária (em anos) } \\
\hline $60-69$ & 55 & 22,0 & 8 & 3,5 & 12 & 5,0 & 12 & 4,5 \\
\hline 70 e + & 50 & 25,5 & 4 & 2,3 & 4 & 2,3 & 2 & 1,2 \\
\hline Valor $\mathrm{p}$ & & 0,261 & & 0,364 & & 0,213 & & 0,054 \\
\hline
\end{tabular}

*Vôlei, futebol, bicicleta e outros. 
madas 18. No entanto, a vantagem da utilização de questionários auto-respondidos ou aplicados por entrevistador está na maior operacionalidade e no baixo custo quando comparado a outros métodos 3,18,19.

Há ainda, como fator limitante do estudo, o fato de que os dados avaliados não foram obtidos de um inquérito específico sobre atividade física de lazer e sim de um inquérito que contemplou diversos temas, entre eles, a atividade física, o que impossibilita o desenvolvimento e aprofundamento de algumas análises por falta de detalhamento de informações.

A obtenção de dados referidos de peso e altura para calcular o IMC tem sido bastante utilizada e considerada válida para estudos populacionais 14 . Entretanto, Silveira et al. 20 verificaram que estas informações são menos válidas quando obtidas de pessoas idosas. Os autores consideram que os idosos conferem o peso e a altura com menor freqüência, levando ao desconhecimento dos valores reais dessas medidas e, conseqüentemente, à pior estimativa do IMC para esta faixa etária em comparação aos mais jovens. No presente estudo, pessoas com sobrepeso apresentaram prevalência de sedentarismo inferior às de peso normal e às obesas, mas a significância estatística desaparece quando a razão de prevalências é ajustada pelas demais variáveis do modelo de regressão múltipla.

Um ponto importante a ser considerado na interpretação dos resultados deste estudo é o viés de causalidade reversa, ou seja, o desenho de corte transversal não permite identificar se os fatores antecederam ou sucederam à presença do sedentarismo. Isso é especialmente válido quando se analisa a associação do sedentarismo com tabagismo e com transtorno mental comum.

Encontrou-se que 70,9\% dos idosos de Campinas não realizam qualquer atividade física durante o lazer. Esta prevalência foi um pouco inferior à encontrada para a população da mesma faixa etária residente em Bambuí (79,5\%), em que foi indagado se nos últimos trinta dias, durante o lazer, havia sido realizada caminhada ou qualquer outro tipo de exercício por pelo menos trinta minutos por semana ${ }^{21}$, e ao observado para a população residente em Salvador $(77,7 \%)$, em que foram considerados sedentários aqueles que informaram não participar de qualquer atividade física no momento de lazer em uma semana habitual 22. Outro estudo, realizado nas regiões Nordeste e Sudeste do Brasil, encontrou que apenas $8,7 \%$ dos homens e $6,8 \%$ das mulheres de 60 anos e mais referiram praticar alguma atividade física de lazer, sendo esta definida como a atividade física realizada por no mínimo trinta minutos, ao menos uma vez na semana 23.
Pesquisa realizada em 15 países da União Européia encontrou prevalência de sedentarismo da ordem de $26,4 \%$, em idosos com 65 anos ou mais, portanto muito inferior à prevalência observada no presente estudo. Mas, naquela pesquisa, foram considerados como sedentários os indivíduos que referiram não praticar qualquer atividade física durante o tempo de lazer além de despender mais de seis horas semanais em atividade sentada 3 .

No presente estudo a prevalência de mulheres sedentárias mostrou-se superior à dos homens, semelhante ao observado por Monteiro et al. 23 em pessoas com 50 anos ou mais e por Pitanga \& Lessa 22 em idosos de 60 anos ou mais. Em pesquisa realizada no Peru, que abrangeu a região metropolitana de Lima e área urbana de municípios com mais de 2 mil habitantes, foi observada também diferença estatisticamente significativa entre o sedentarismo de homens $(47,7 \%)$ e de mulheres $(71,7 \%)$ com 60 anos e mais. Diferentes padrões sociais, culturais e de adesão à prática de esporte explicariam as diferenças de sedentarismo observadas entre homens e mulheres 24 .

Idosos de menor nível sócio-econômico, nível avaliado por renda ou escolaridade, são mais sedentários no lazer. Diversas pesquisas verificaram que a atividade física durante o lazer está relacionada positivamente ao nível sócio-econômico 2,17,25, enquanto o padrão inverso é encontrado quando se considera a atividade física global, ou seja, quando a atividade ocupacional é incluída na análise 26,27 . Indivíduos com menor nível de escolaridade tendem a adotar, com mais freqüência, hábitos de vida prejudiciais à saúde, por disporem de menor acesso à informação e às condições de vida que propiciam a incorporação de comportamentos considerados saudáveis 22,28 .

A presente pesquisa permitiu verificar que os idosos fumantes apresentaram-se mais sedentários que os não-fumantes, similarmente ao observado em alguns outros estudos 3,25,29, o que aponta para a concomitância de comportamentos não saudáveis em alguns subgrupos da população.

Verificou-se associação positiva entre transtorno mental comum e sedentarismo no lazer. Como explicitado anteriormente, o desenho de estudo não permite o esclarecimento da relação de causa-efeito e a literatura traz relatos tanto apontando que o sedentarismo contribui para o risco de depressão, de ansiedade e de prejuízos para a saúde mental 30 , assim como achados de maior prevalência de inatividade física entre pessoas com depressão autopercebida 17. Também existem evidências das repercussões psíquicas 
positivas decorrentes da prática de atividade física sobre a depressão, a ansiedade e a melhora do bem-estar psíquico ${ }^{31}$. Em revisão sistemática da literatura, Peluso \& Andrade ${ }^{6}$ apontam que a atividade física seria responsável por atuar diretamente nos fatores psicológicos (distração, auto-eficácia e interação social) e nos fatores fisiológicos (aumento da transmissão sináptica das monoaminas, que supostamente funcionaria como as drogas antidepressivas e o efeito inibitório da morfina endógena no sistema nervoso central) que resultariam, conseqüentemente, na melhora da ansiedade e do humor após a prática de atividade física.

O estudo permitiu estimar que $23,5 \%$ dos idosos de Campinas fazem caminhadas ao menos uma vez por semana, sendo esta a modalidade mais comum de exercício entre os idosos. Este resultado é semelhante ao encontrado para a população americana de 65 anos e mais em que a prevalência de caminhada é $23,6 \% 1$. Pesquisadores mencionam a caminhada como a atividade mais comum praticada em países desenvolvidos e em desenvolvimento ${ }^{32}$, acessível e popular entre homens e mulheres e que pode ser praticada em diferentes intensidades e em qualquer lugar 2,9,26. Esta modalidade é também destaque nos programas de incentivo à prática de atividade física e mais indicada para pessoas com mais idade por ser considerada uma atividade leve ou moderada, de baixo impacto e que envolve grandes grupos musculares 5,26.

As análises mostraram que idosos de melhor nível sócio-econômico (renda e escolaridade) apresentam prevalência de caminhar superior à observada para idosos com pior condição econômica. Estas disparidades podem indicar que os indivíduos mais pobres tenham menos conhecimento sobre os benefícios da atividade física e vivam em contextos menos favoráveis para a incorporação destas atividades de lazer na sua rotina 33 .

Verificou-se que ginástica/musculação foi a segunda modalidade mais praticada, principalmente entre as mulheres, assim como havia sido observado em outros estudos 5,23,24. Deve-se lembrar que, entre os idosos, esta modalidade é difundida pelos benefícios na prevenção na perda de massa óssea, bem como no controle e tratamento da osteoporose 34 . Em relação à natação ou hidroginástica, são modalidades bastante recomendadas para os idosos, por serem de baixo impacto e com menor risco de lesões músculo-esqueléticas e especialmente indicada para aqueles que apresentam doenças articulares degenerativas, como a osteoartrose 35 .

Os achados desta pesquisa, em que o sedentarismo no lazer destaca-se entre os idosos do sexo feminino, os que fumam, os que apresentam transtorno mental comum e os que pertencem a segmentos sócio-econômicos menos favorecidos, apontam que os programas voltados a estimular a prática de exercício físico no lazer precisam atuar integrados a outras ações de promoção da saúde, como as de controle do tabagismo. Sugere, também, que os profissionais de saúde precisam estar atentos em identificar a presença de transtorno mental comum em idosos, pois aqueles com o transtorno terão maior dificuldade em aderir às práticas de exercício e, por outro lado, seriam os idosos que muito se beneficiariam, inclusive nos aspectos emocionais, com o desenvolvimento destas práticas. Os resultados indicariam, ainda, que as políticas públicas voltadas à promoção de qualidade de vida, devem privilegiar, em especial, os segmentos socialmente mais desfavorecidos evitando um crescimento das desigualdades sociais na morbimortalidade e assegurando maior eqüidade na adoção de comportamentos favoráveis à saúde. 


\section{Resumo}

O objetivo deste estudo foi conhecer a prevalência de sedentários no lazer (que referem não praticar nenhum exercício físico no lazer ao menos uma vez por semana) em idosos de Campinas, São Paulo, Brasil, segundo fatores demográficos e sócio-econômicos, outros comportamentos relacionados à saúde e à presença de morbidades. Trata-se de um estudo transversal, de base populacional, com amostragem em múltiplos estágios. A análise dos dados levou em conta o desenho amostral. A prevalência de sedentários foi 70,9\%, sendo que as razões de prevalências foram significativamente maiores que um para os idosos de menor renda $(1,31$ : 1,11-1,55), tabagistas (1,39: 1,23-1,57), com transtorno mental comum $(1,20: 1,04-1,39)$ e do sexo feminino (1,16: 1,00-1,35). A prevalência de caminhada foi $23,5 \%$, seguida por ginástica ou musculação $(3,8 \%) e$ por natação ou hidroginástica (3,6\%). Os resultados apontam para a necessidade do desenvolvimento de ações globais com respeito aos comportamentos relacionados à saúde. Atenção especial deve ser dada aos idosos do sexo feminino, àqueles com transtorno mental comum e aos de menor nível sócio-econômico a fim de garantir eqüidade em relação às práticas de promoção da saúde.

Atividades de Lazer; Exercício; Idoso; Conduta de Saúde; Promoção da Saúde

\section{Referências}

1. Centers for Disease Control and Prevention. Compliance with physical activity recommendations by walking for exercise - Michigan, 1996 and 1998. MMWR Morb Mortal Wkly Rep 2000; 49:560-5.

2. Eyler AA, Browson RC, Bacak SJ, Housemann RA. The epidemiology of walking for physical activity in the United States. Med Sci Sports Exerc 2003; 35:1529-36.

3. Varo JJ, Martínez-González MA, Irala-Estévez J, Kearney J, Gibney M, Martínez JA. Distribution and determinants of sedentary lifestyles in the European Union. Int J Epidemiol 2003; 32:138-46.

4. Blair SN. Physical activity, epidemiology, public health and the American College of Sports Medicine. Med Sci Sports Exerc 2003; 35:1463.

5. Salles-Costa R, Heilborn ML, Werneck GL, Faerstein E, Lopes CS. Gênero e prática de atividade física de lazer. Cad Saúde Pública 2003; 19 Suppl 2: S325-33.

\section{Colaboradores}

M. P. A. Zaitune realizou a proposta do artigo, a revisão de literatura, a análise dos dados e a redação do artigo. M. B. A. Barros orientou a proposta do artigo, a análise dos dados e a redação do artigo. M. B. A. Barros, C. L. G. César, L. Carandina e M. Goldbaum coordenaram a pesquisa de campo e contribuíram na revisão do artigo.

\section{Agradecimentos}

À Fundação de Amparo à Pesquisa do Estado de São Paulo (Projeto de Políticas Públicas, processo no. 88/14099) e à Secretaria Estadual de Saúde de São Paulo pelo financiamento do trabalho de campo. À Secretaria de Vigilância em Saúde do Ministério da Saúde pelo suporte financeiro para a análise dos dados através do Centro Colaborador em Análise de Situação de Saúde da Universidade Estadual de Campinas; à Coordenação de Aperfeiçoamento de Pessoal de Nível Superior pela bolsa de mestrado recebida pela autora principal.
6. Peluso MAM, Andrade LHSG. Physical activity and mental health: the association between exercise and mood. Clinics 2005; 60:61-70.

7. Santos LD, Teixeira-Salmela LF, Lelis FO, Lobo MB. Eficácia da atividade física na manutenção do desempenho funcional do idoso: uma revisão da literatura. Fisioter Bras 2001; 2:169-76.

8. Organização Mundial da Saúde. Envelhecimento ativo: uma política de saúde. Brasília: Organização Pan-Americana da Saúde/Organização Mundial da Saúde; 2005.

9. Giles-Corti B, Donavan RJ. Relative influences of individual, social environmental and physical environmental correlates of walking. Am J Public Health 2003; 93:1583-9.

10. Gomes VB, Siqueira KS, Sichieri R. Atividade física em uma amostra probabilística da população do município do Rio de Janeiro. Cad Saúde Pública 2001; 17:969-76. 
11. César CLG, Carandina L, Alves MCGP, Barros MBA, Goldbaum M. Saúde e condição de vida em São Paulo: inquérito multicêntrico de saúde no estado de São Paulo (ISA-SP). São Paulo: Faculdade de Saúde Pública, Universidade de São Paulo; 2005.

12. Bisson J, Nadeau L, Demers A. The validity of the CAGE scale to screen for heavy drinking and drinking problem in a general population survey. Addiction 1999; 94:715-22.

13. World Health Organization. A user's guide to the Self Reporting Questionnaire. Geneva: World Health Organization; 1994.

14. Barros MBA. Obesidade. In: César CLG, Carandina L, Alves MCGP, Barros MBA, Goldbaum M, organizadores. Saúde e condição de vida em São Paulo: inquérito multicêntrico de saúde no estado de São Paulo (ISA-SP). São Paulo: Faculdade de Saúde Pública, Universidade de São Paulo; 2005. p. 15162.

15. Latorre MRDO. Medidas de risco e regressão logística. In: Massad E, Ortega NRS, Silveira PSP, Menezes RX, organizadores. Métodos quantitativos em medicina. São Paulo: Manole; 2004. p. 337-49.

16. Secretaria de Políticas de Saúde, Ministério da Saúde. Programa Nacional de Promoção da Atividade Física "Agita Brasil": atividade física e sua contribuição para a qualidade de vida. Rev Saúde Pública 2002; 36:254-6.

17. Yancey AK, Wold CM, McCarthy WJ, Weber MD, Lee B, Simon PA, et al. Physical inactivity and overweight among Los Angeles county adults. Am J Prev Med 2004; 27:146-52.

18. Motl RW, McAuley E, DiStefano C. Is social desirability associated with self-reported physical activity? Prev Med 2005; 40:735-9.

19. Bowles HR, FitzGerald SJ, Morrow Jr. JR, Jackson AW, Blair SM. Construct validity of self-reported historical physical activity. Am J Epidemiol 2004; 160:279-86

20. Silveira EA, Araújo CL, Gigante DP, Barros AJD, Lima MS. Validação do peso e altura referidos para o diagnóstico do estado nutricional em uma população de adultos no Sul do Brasil. Cad Saúde Pública 2005; 21:235-45.

21. Lima-Costa M, Barreto S, Uchoa E, Firmo J, Vidigal P, Guerra E. The Bambuí Health and Aging Study (BHAS): prevalence of risk factors and use of preventive health care services. Rev Panam Salud Pública 2001; 9:219-27.

22. Pitanga FJG, Lessa I. Prevalência e fatores associados ao sedentarismo no lazer em adultos. Cad Saúde Pública 2005; 21:870-7.
23. Monteiro CA, Conde WL, Matsudo SM, Matsudo VR, Bonseñor IM, Lotufo PA. A descriptive epidemiology of leisure-time physical activity in Brazil, 1996-1997. Rev Panam Salud Pública 2003; 14:24654.

24. Seclén-Palacín JA, Jacoby ER. Factores sociodemográficos y ambientales asociados con la actividad física deportiva en la población urbana del Peru. Rev Panam Salud Pública 2003; 14:255-64.

25. Crespo CJ, Smit E, Carter-Pokras O, Andersen R. Acculturation and leisure-time physical inactivity in Mexican Americans adults: results from NHANES III, 1988-1994. Am J Public Health 2001; 91:1254-7.

26. Hallal PC, Victora CG, Wells JC, Lima RC. Physical inactivity: prevalence and associated variables in Brazilian adults. Med Sci Sports Exerc 2003; 35:1894-900.

27. Hallal PC, Azevedo MR, Reichert FF, Siqueira, FV, Araújo CLP, Victora, CG. Who, when, and how much? Epidemiology of walking in a middle-income country. Am J Prev Med 2005; 28:156-61.

28. Lima-Costa MF. Estilos de vida e uso de serviços preventivos de saúde entre adultos filiados ou não a plano privado de saúde (inquérito de saúde de Belo Horizonte). Ciênc Saúde Coletiva 2004; 9:85764.

29. Paavola M, Vartiainen E, Haukkala A. Smoking, alcohol use, and physical activity: a 13-year longitudinal study ranging from adolescence into adulthood. J Adolesc Health 2004; 35:238-44.

30. Centers for Disease Control and Prevention. Monthly estimates of leisure-time physical inactivity - United States, 1994. MMWR Morb Mortal Wkly Rep 1997; 46:393-7.

31. Palmer C. Exercise as a treatment for depression in elders. J Am Acad Nurse Pract 2005; 17:60-6.

32. Hallal PC, Matsudo SM, Matsudo VKR, Araújo TL, Andrade DR, Bertoldi AD. Physical activity in adults from two Brazilian areas: similarities and differences. Cad Saúde Pública 2005; 21:573-80.

33. Domingues MR, Araújo CLP, Gigante DP. Conhecimento e percepção sobre exercício físico em uma população adulta urbana do sul do Brasil. Cad Saúde Pública 2004; 20:204-15.

34. Brown JP, Josse RG. Clinical practice guidelines for the diagnosis and management of osteoporosis in Canada. Can Med Assoc J 2002; 167 Suppl 10:S134.

35. Nicholas JJ. Physical modalities in rheumatological rehabilitation. Arch Phys Med Rehabil 1994; 75:994-1001.

Recebido em 16/Mar/2006

Versão final reapresentada em 28/Jul/2006

Aprovado em 20/Dez/2006 